\title{
Amino Acid Deprivation Inhibits TORC1 Through a GTPase-Activating Protein Complex for the Rag Family GTPase Gtr1
}

\author{
Nicolas Panchaud, ${ }^{*}$ Marie-Pierre Péli-Gulli, ${ }^{*}$ Claudio De Virgilio ${ }^{\dagger}$ \\ The Rag family of guanosine triphosphatases (GTPases) regulates eukaryotic cell growth in response \\ to amino acids by activating the target of rapamycin complex 1 (TORC1). In humans, this pathway is \\ often deregulated in cancer. In yeast, amino acids promote binding of GTP (guanosine 5'-triphosphate) \\ to the Rag family GTPase Gtr1, which, in combination with a GDP (guanosine diphosphate)-bound \\ Gtr2, forms the active, TORC1-stimulating GTPase heterodimer. We identified Iml1, which functioned \\ in a complex with Npr2 and Npr3, as a GAP (GTPase-activating protein) for Gtr1. Upon amino acid \\ deprivation, Iml1 transiently interacted with Gtr1 at the vacuolar membrane to stimulate its intrinsic \\ GTPase activity and consequently decrease the activity of TORC1. Our results delineate a potentially \\ conserved mechanism by which the Iml1, Npr2, and Npr3 orthologous proteins in humans may sup- \\ press tumor formation.
}

\section{INTRODUCTION}

The structurally and functionally conserved target of rapamycin complex 1 (TORC1) is a central element of a signaling pathway that integrates various hormonal, growth factor, energy, and nutritional cues to coordinate growth, metabolism, and aging $(1,2)$. In yeast, activated TORC1 propagates nutrient signals mainly through the AGC protein kinase Sch9 and Tap42, a regulator of type $2 \mathrm{~A}$ (and type $2 \mathrm{~A}$-like) protein phosphatases, to favor anabolic processes and inhibit catabolic processes and stress response programs $(3,4)$. A primordial TORC1 input signal that cannot be compensated for by any other stimulus is provided by amino acids, in particular branched-chain amino acids such as leucine. The mechanism through which TORC1 senses amino acids requires the highly conserved Rag family of guanosine triphosphatases (GTPases), which function in heterodimeric complexes that combine RagA or RagB with RagC or RagD in higher eukaryotes, or Gtr1 with Gtr2 in yeast (5-7). The Rag or Gtr heterodimers are asymmetrically loaded with GTP (guanosine $5^{\prime}$-triphosphate) and GDP (guanosine diphosphate) and can stimulate TORC1 in response to amino acids when RagA, RagB, or Gtr1 is bound to GTP. The mechanistic details of how amino acids modulate the configuration of the Rag or Gtr heterodimers are still a matter of debate, but likely involve both specific guanine nucleotide exchange factors (GEFs) and GTPase-activating proteins (GAPs). It has been proposed that lysosomal amino acids, through an inside-out mechanism that requires the vacuolar $\mathrm{H}^{+}$-ATPase $\left(\mathrm{H}^{+}\right.$-translocating adenosine triphosphatase) (v-ATPase) (8), stimulate the GEF activity of the Ragulator complex toward $\operatorname{RagA}$ or $\operatorname{RagB}(9,10)$. Whether this represents an ancestral mode of the regulation of Rag GTPase activity remains to be determined because yeast cells do not express apparent orthologs of critical components of the Ragulator complex and may promote GTP loading of Gtr1 through the GEF Vam6 $(6,11)$. Amino acid signaling may also involve leucyl-tRNA (transfer RNA) synthetase, which acts as a leucine sensor that promotes the active conformation of the Rag heterodimers $(12,13)$. A putative GAP that suppresses the activity of RagA, RagB, or Gtrl after amino

Department of Biology, Division of Biochemistry, University of Fribourg, $\mathrm{CH}-1700$ Fribourg, Switzerland.

*These authors contributed equally to this work.

+Corresponding author. E-mail: claudio.devirgilio@unifr.ch acid deprivation has yet to be identified. Here, we report on our discovery that in yeast, Iml1 functions in an Npr2/3-containing complex as a GAP for Gtr1 to decrease the activity of TORC1 and consequently growth after amino acid deprivation.

\section{RESULTS}

A genome-wide screen for regulators that inhibit TORC1 in response to amino acid deprivation identified the yeast proteins Npr2 and Npr3 (14), which together with Iml1 assemble into a complex that is embedded within the larger, vacuolar membrane-associated SEA complex (SEAC) (15). The Iml1-Npr2-Npr3 complex has also been proposed to promote autophagy, although independently of TORC1 (16). To clarify the role of SEAC in TORC1 regulation, we assessed TORC1 activity in cells lacking individual components of the SEAC, all of which grew normally at $30^{\circ} \mathrm{C}$ and responded properly to leucine starvation in terms of phosphorylation of the eukaryotic translation initiation factor $2 \alpha$ (eIF2 $\alpha$ ), a sensitive indicator of the presence of uncharged tRNAs that stimulate the eIF $2 \alpha$ kinase Gen2 (17) (fig. S1, A and B). Loss of Iml1, Npr2, or Npr3, but not of Sea2, Sea3, or Sea4, resulted in increased TORC1 activity, as assessed by phosphorylation of Sch9 (Fig. 1, A and B). Together with the observation that the concomitant loss either of $\mathrm{Npr} 2$ and $\mathrm{Npr} 3$ or of $\mathrm{Npr} 2$, Npr3, and Iml1 stimulated TORC1 activity to similar extents (Fig. 1C and fig. S2), these data indicate that Npr2, Npr3, and Iml1 share a common biological function in inhibiting TORC1. In contrast, Sea2, Sea3, and Sea4 appeared to redundantly attenuate the TORC1 inhibitory properties of the Iml1-Npr2-Npr3 complex because combined loss of Sea2, Sea3, and Sea4 (Sea2/3/4) decreased TORC1 activity in an Iml1-, Npr2-, or Npr3-dependent manner (Fig. 1C and fig. S2). To further dissect the roles of the individual components of the Iml1-Npr2-Npr3 complex, we overproduced Iml1, Npr2, Npr3, or Npr2 together with Npr3 and examined the corresponding effects on TORC1 activity in wild-type, iml1 $1 \Delta, n p r 2 \Delta$, and $n p r 3 \Delta$ strains. Overproduction of Imll bypassed the requirement for Npr2 or Npr3 to decrease the activity of TORC1, but not vice versa (Fig. 1D). Consistent with these genetic data, Iml1 did not require Npr2/3 or Sea $2 / 3 / 4$ to be recruited to the vacuolar membrane (Fig. 1, E and F), whereas Npr2 and Npr3 depended on each other and on Iml1 for their localization to the vacuolar membrane. This led us to speculate that the 
A

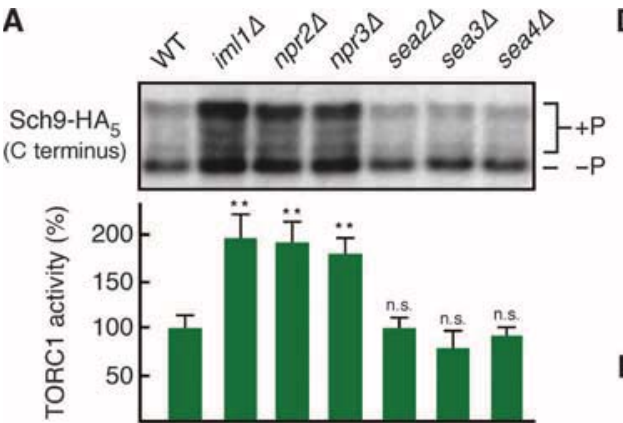

B

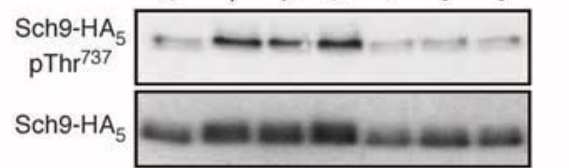

C

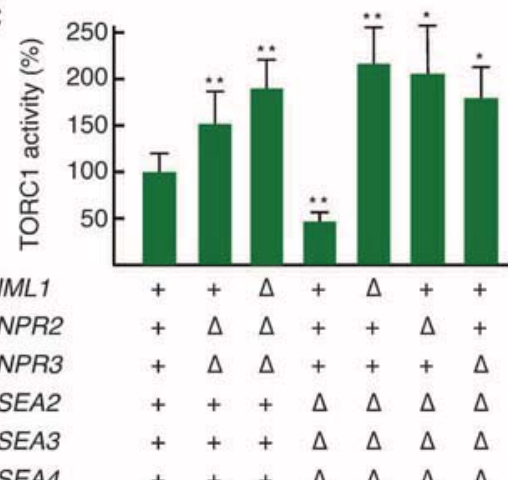

。

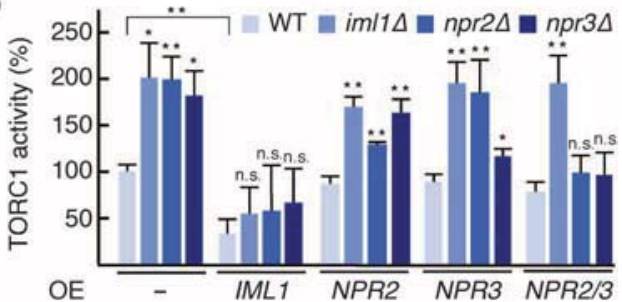

E

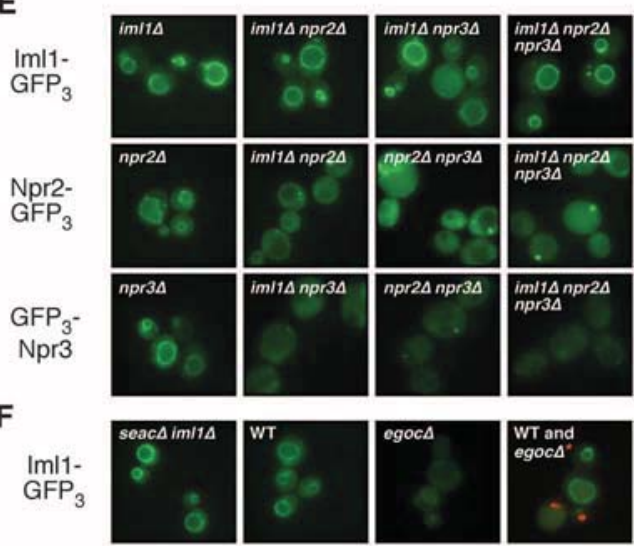

Fig. 1. Vacuolar membrane-associated Iml1-Npr2-Npr3 complex inhibits TORC1. (A and B) Loss of Iml1, Npr2, or Npr3, but not of Sea2, Sea3, or Sea4, causes an increase in TORC1 activity. (A) Immunoblots detecting the extent of phosphorylation in the C terminus of Sch9 were used to quantify TORC1 activity [the ratio of hyperphosphorylated (+P)/hypophosphorylated (-P) Sch9] (25). The values were normalized to those for wild-type (WT) cells and presented in the bar graph as means $\pm \mathrm{SD}$ ( $n=3$ independent experiments). (B) Similar results for TORC1 activities (ratio of $\mathrm{pThr}^{737}$ /total full- length Sch9) were obtained by using specific antibodies recognizing the phosphorylated $\mathrm{Thr}^{737}\left(\mathrm{pThr}^{737}\right.$ ) of Sch9 (26), a major TORC1 target in yeast (25). One representative immunoblot from three independent experiments is shown. (C and D) Effects on TORC1 activity of various combinations of individual deletions ( $\Delta$ ) of SEAC subunit-encoding genes (C) and of overexpression (OE) of IML1 or of NPR2 and NPR3 (individually or in combination) in different mutant backgrounds (D). Data are means $\pm S D$ from three independent experiments. (E and F) WT or mutant strains expressing the indicated functional green fluorescent protein (GFP) fusion proteins from their own promoter were analyzed by fluorescence microscopy during exponential growth. (F) egocs and

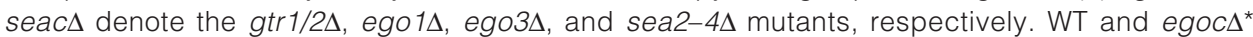
(expressing the nuclear marker Hhf2-RFP) cells were mixed to directly compare Iml1-GFP 3 signals at the vacuolar membrane. Representative images from three independent experiments are shown. ${ }^{*} P<0.01 ;{ }^{*} P<0.05$; n.s., not significant, compared to the respective WT control using Student's $t$ test ( $P$ values are Holm-Bonferroni-adjusted).

functional roles within the Iml1-Npr2-Npr3 complex may be partitioned into a catalytic role for Iml1 and structural and/or regulatory roles for $\mathrm{Npr} 2$ or Npr3.

Our cell biological analyses revealed that Imll required the presence of the EGO complex [constituting Gtr1 and Gtr2 and their vacuolar membrane anchors Egol and Ego3 (18)] to be efficiently localized at the vacuolar membrane (Fig. 1F). This suggested that Iml1 may regulate TORC1 through the Gtr1-Gtr2 heterodimer. Consistent with this idea, the absence of Gtr1 or Gtr2 (or of the TORC1 subunit Tco89; Fig. 2A) or the expression of signaling-compromised
$\mathrm{Gtrl}^{\mathrm{S} 20 \mathrm{~L}}$ (which has low affinity for nucleotides) or GTP-locked Gtr2 ${ }^{\text {Q66L }}$ (Fig. 2B) prevented the increase in activation of TORC1 in imll $\Delta$ cells. Increased TORC1 activation in $n p r 2 \Delta$ and $n p r 3 \Delta$ cells was also dependent on Gtr1 (Fig. 2A). Moreover, expression of the GTP-locked, signalingcompetent $\mathrm{Gtrl}^{\mathrm{Q} 65 \mathrm{~L}}$ allele and loss of Iml1, both individually and in combination, stimulated TORC1 to a similar extent, indicating that Imll may in fact specifically function upstream of and inhibit Gtr1 (Fig. 2B). Two additional observations support this notion: (i) TORC1 activity remained sensitive to loss or overproduction of $\mathrm{Iml1}$ in the presence of $\mathrm{Gtr} 2^{\mathrm{S} 23 \mathrm{~L}}$, which has low affinity for nucleotides (Fig. 2, $\mathrm{B}$ and $\mathrm{C}$ ), but (ii) was largely refractory

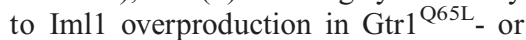
$\mathrm{Gtr}^{\mathrm{S} 2 \mathrm{LL}}$-expressing cells (Fig. 2C). Last, as predicted by a model in which the Iml1Npr2-Npr3 complex decreases the activity of Gtr1 after amino acid deprivation, loss of Iml1, or of Npr2 and Npr3 (individually or in combination), like overexpression of $\mathrm{Gtr} 1^{\mathrm{Q} 65 \mathrm{~L}}$, rendered TORC1 activity partially resistant to leucine starvation (Fig. 2D and fig. S3).

Our genetic epistasis analyses led us to examine whether Imll interacted with Gtr1 in cells. Imll specifically bound Gtrl in the presence, but substantially less in the absence, of Npr2 and Npr3 (Fig. 3A and fig. S4). Moreover, although GFP-fused variants of Iml1, Npr2, and Npr3, like EGOC and TORC1 (6), all localized to the vacuolar membrane both before and after leucine deprivation (Fig. 3B), bimolecular fluorescence complementation (BiFC) analysis revealed that leucine deprivation stimulated the Iml1-Gtr1 interaction specifically at the vacuolar membrane (Fig. 3C). This was also readily observable in coimmunoprecipitation experiments in which leucine deprivation transiently stimulated the Iml1-Gtr1 interaction, but not the constitutively strong $\mathrm{Iml1}-\mathrm{Gtr} 1^{\mathrm{Q} 65 \mathrm{~L}}$ interaction (Fig. 3D). To explore whether Iml1 regulated the GTP-loading status of Gtrl in cells, we made use of the fact that the TORC1 subunit Kog1 binds preferentially the GTP-bound form of Gtrl (6); hence, the amount of Gtr1-associated $\operatorname{Kog} 1$ can be used as a proxy for the relative amount of Gtr1 ${ }^{\text {GTP }}$ within cells. We observed that Imll overproduction reduced the Kog1-Gtr1 interaction, but not the Kog1-Gtr1 ${ }^{\text {Q65L }}$ interaction (Fig. 3E), which implicates Imll as a potential GAP for Gtr1. In accordance with this notion, the binding of purified Iml1 to Gtr1 was enhanced by the presence of the nonhydrolyzable GTP analog GTP $\gamma \mathrm{S}$ or of GDP$\mathrm{AlF}_{\mathrm{x}}$, which is a structural mimic of the transition state in the hydrolysis reaction by GTPases (Fig. 3F) (19) and identifies interactions of GTPases with their cognate GAPs (20). 

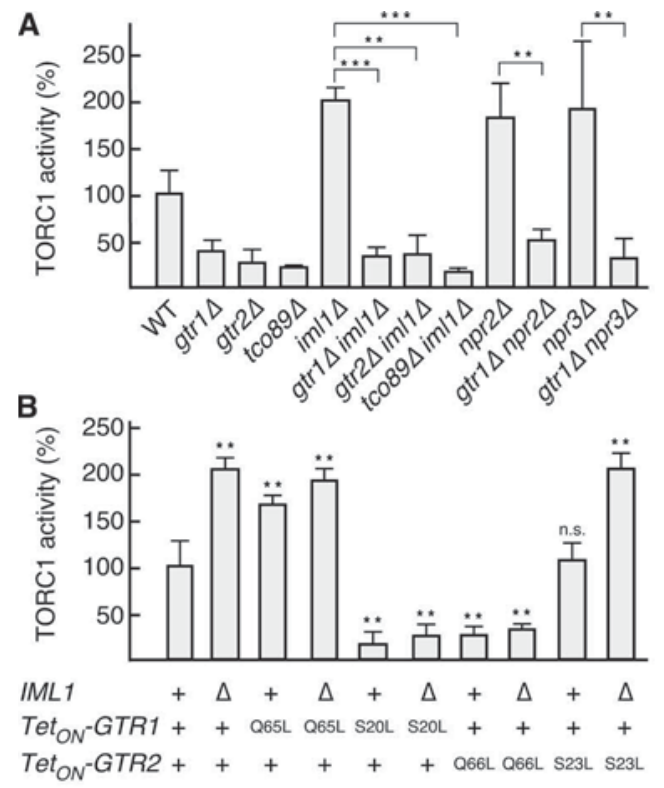

C
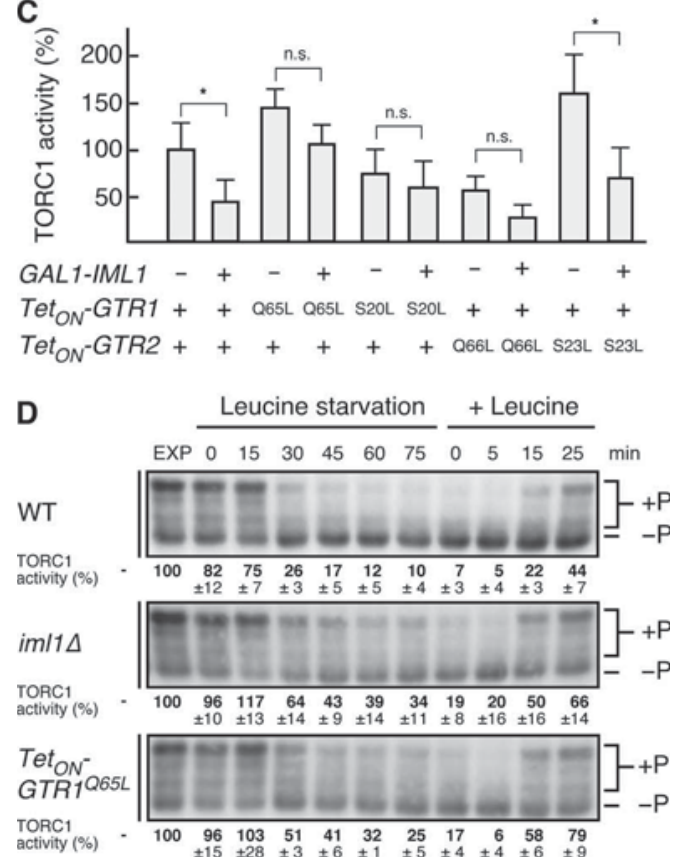

Fig. 2. The Iml1-Npr2-Npr3 complex controls TORC1 activity through the Rag GTPase heterodimer Gtr1-Gtr2. (A) Increased TORC1 activity observed in the absence of Iml1, Npr2, or Npr3 requires the presence of Gtr1 or Gtr2 (means \pm SD from three independent experiments). (B and C) Effects on TORC1 activity of loss (B) and overproduction of Iml1 (C) in

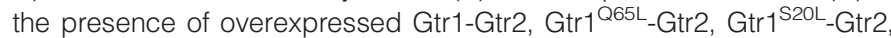
Gtr1-Gtr2 $^{\mathrm{Q} 66 \mathrm{~L}}$, or Gtr1-Gtr2 ${ }^{\mathrm{S} 23 \mathrm{~L}}$ heterodimers (means $\pm \mathrm{SD}$ from three independent experiments). (D) Loss of Iml1 or expression of Gtr $1^{\mathrm{Q} 65 \mathrm{~L}}$ renders TORC1 activity partially insensitive to leucine starvation (assayed as in Fig. 1A). Numbers are means \pm SD from three independent experiments; one representative immmunoblot is shown. ${ }^{* *} P<0.001$; ${ }^{* \star} P<0.01$; ${ }^{*} P<0.05$; n.s. not significant, compared to the indicated control in $(A)$ and $(C)$ or to WT in (B) using Student's $t$ test ( $P$ values are Holm-Bonferroni-adjusted).
To verify our assumption that Imll has GAP activity toward Gtrl, we performed in vitro GAP assays with purified Imll and Gtrl proteins. In a concentration-dependent manner, Imll stimulated the rate of GTP hydrolysis by Gtr1 to a greater extent than that by Gtr2 or the unrelated Rho GTPase Cdc42 (Fig. 4, A and B). Analyses of truncated Iml1 versions further allowed us to specify a conserved domain in Iml1 that was required for proper TORC1 inhibition (Fig. 4C) and was sufficient to stimulate the GTP hydrolysis rate by Gtr1 in vitro (Fig. 4D). In single-turnover GAP assays, this domain, like full-length Iml1, substantially accelerated the catalytic rate of Gtr1-mediated GTP hydrolysis and was therefore coined the Iml1 GAP domain (Iml1 ${ }^{\text {GAP }}$; Fig. 4, C and E). GAPs often supply a catalytic amino acid residue such as an arginine (Arg), glutamine (Gln), or aspartate (Asp) into the active site of their GTPases (19), which prompted us to carry out an alanine scanning approach. We identified $\mathrm{Arg}^{943}$, which is located within Iml1 ${ }^{\mathrm{GAP}}$ (Fig. 4C), as critical for the GAP activity of Iml1 in vitro (Fig. 4, D and E). The R943A mutation partially, but significantly, reduced the TORC1 inhibitory function of Iml1 in cells (figs. S3 and $\mathrm{S} 5, \mathrm{~A}$ and $\mathrm{B}$ ). Together with the observation that $\mathrm{GDP}_{-} \mathrm{AlF}_{\mathrm{x}}$ promoted the in vitro interaction of Gtrl with wild-type Imll and Iml1 ${ }^{\mathrm{R} 943 \mathrm{~A}}$ to the same extent (fig. S6), these data suggest that $\mathrm{Arg}^{943}$ is a catalytically, rather than structurally, important residue within the Gtr1 GAP Imll. However, detailed structural analyses will be required to assess whether $\mathrm{Arg}^{943}$ in Iml1 interacts with the catalytic domain of Gtr1. Although Gtr1-Gtr2 ${ }^{\mathrm{Q} 66 \mathrm{~L}}$ or Gtr1$\mathrm{Gtr}^{\mathrm{S} 23 \mathrm{~L}}$ heterodimer formation per se stimulated the intrinsic GTPase activity of Gtr1 16- or 128-fold, respectively, Iml1 exhibited GAP activity toward both monomeric and heterodimeric Gtr1 in a comparable range (Fig. 4F). Thus, Gtr1-mediated GTP hydrolysis in Gtr GTPase heterodimers appears to be controlled synergistically by both the GDP/GTP loading status of Gtr2 and the activity of Iml1. Finally, supporting the evolutionary conservation of our findings, we found that the human Iml1 ortholog DEPDC5 could partially rescue the TORC1 inhibition defect in imlls cells (Fig. 4G).

\section{DISCUSSION}

In association with $\mathrm{Npr} 2$ and $\mathrm{Npr} 3, \mathrm{Iml} 1$ functions as a GAP for Gtr1 to restrain it from activating TORC1 specifically in response to amino acid deprivation. Iml1, Npr2, and Npr3 have orthologs in humans (DEPDC5, NPRL2, and NPRL3, respectively), of which NPRL2 has previously been classified as a suppressor of various tumors (21-23). In addition, the identification of overlapping homozygous deletions encompassing DEPDC5 (and two other genes) in two cases of glioblastoma suggests that loss of DEPDC5 may contribute to the development of cancer (24). Because DEPDC5 could partially complement the loss of Imll in yeast, we speculate that the Iml1Npr2-Npr3 complex may play an evolutionarily conserved role in suppressing Rag-mediated activation of TORC1, thereby contributing to the suppression of human tumor formation.

\section{MATERIALS AND METHODS}

\section{Strains, growth conditions, and plasmids}

Unless stated otherwise, prototrophic strains were pregrown overnight in synthetic dropout medium $(0.17 \%$ yeast nitrogen base, $0.5 \%$ ammonium sulfate, $0.2 \%$ dropout mix, and $2 \%$ glucose). Before each experiment, cells were diluted to an $\mathrm{OD}_{600}$ (optical density at $600 \mathrm{~nm}$ ) of 0.2 and further grown at $30^{\circ} \mathrm{C}$ until they reached an $\mathrm{OD}_{600}$ of 0.8 . For leucine deprivation experiments, strains that were specifically auxotrophic for leucine were grown to an $\mathrm{OD}_{600}$ of 0.8 in synthetic dropout medium with leucine $(0.37 \mathrm{mg} / \mathrm{ml})$, filtered, washed twice, and resuspended in same medium devoid of leucine. For galactose induction, precultures were grown 

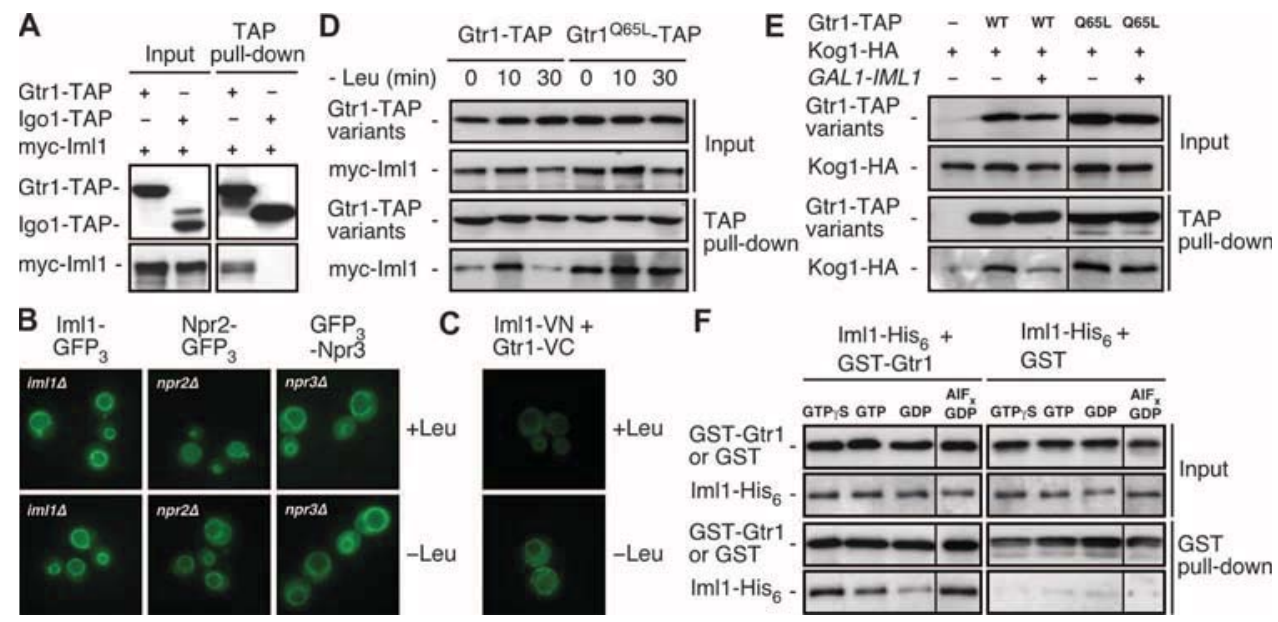

Fig. 3. Leucine inhibits the interaction between Iml1 and Gtr1 at the vacuolar membrane. (A) In exponentially growing WT cells, myc-Iml1 physically interacts with Gtr1-TAP, but not with the control fusion protein Igo1-TAP. Lysates (input) of cells expressing the indicated fusion proteins and TAP pull-down fractions were analyzed by immunoblotting with anti-TAP or anti-myc antibodies. One representative immunoblot from three independent experiments is shown. ( $B$ and $C$ ) Leucine deprivation does not change the vacuolar membrane localization of Iml1-GFP 3 , Npr2-GFP ${ }_{3}$, and GFP 3 -Npr3 (B), but stimulates the interaction between Gtr1 and Iml1 at the vacuolar membrane as assayed through BiFC $(C)$, which allows detection of protein-protein interactions in cells due to reconstitution of the fluorescent Venus protein (29). VN and VC denote N-terminal and C-terminal fragments of Venus, respectively. Representative images are shown from three independent experiments. (D) TAP pull-down analyses indicate that the Iml1-Gtr1 interaction is transiently stimulated after leucine starvation, whereas Iml1 constitutively binds Gtr1 ${ }^{\mathrm{Q} 65 \mathrm{~L}}$. (E) The Gtr1-Kog1 interaction, but not the Gtr1 ${ }^{\mathrm{Q} 65 \mathrm{~L}}-\mathrm{Kog} 1$ interaction, is reduced when IML1 is overexpressed from the GAL1 promoter. (F) Purified Iml1-His, preferentially binds to bacterially expressed GST-Gtr1 preloaded with GTP $\mathrm{S}$, GTP, or $\mathrm{AlF}_{\mathbf{x}}\left(\mathrm{AlF}_{3}\right.$ or $\left.\mathrm{AlF}_{4}^{-}\right)$plus GDP. Data from $(D)$ to $(F)$ are representative immunoblots from three or more independent experiments.

in synthetic medium (SRaf; $0.17 \%$ yeast nitrogen base, $0.5 \%$ ammonium sulfate, $0.2 \%$ dropout mix, $2 \%$ raffinose, and $0.1 \%$ sucrose). Cells were then diluted to an $\mathrm{OD}_{600}$ of 0.2 and further grown in SRaf supplemented with $2 \%$ galactose until they reached an $\mathrm{OD}_{600}$ of 0.8 . Expression of genes under the control of the Tet $t_{O N}$ promoter was induced by adding doxycycline $(5 \mu \mathrm{g} / \mathrm{ml})$ to specified medium. The $S$. cerevisiae strains and plasmids used in this study are listed in tables $\mathrm{S} 1$ and S2, respectively.

\section{TORC1 activity assays}

TORC1 activity was quantified by assessing the phosphorylation of the C-terminal part of hemagglutinin (HA)-tagged Sch $9^{\text {T570, }}$, which contains at least five bona fide TORC1 phosphorylation sites, as described previously $(6,25)$. Briefly, after chemical cleavage with NTCB (2-nitro-5-thiocyanatobenzoic acid), extracts were separated by $7.5 \%$ SDS-polyacrylamide gel electrophoresis (PAGE), and membranes were probed with anti-HA antibodies (12CA5) and anti-mouse immunoglobulin $\mathrm{G}(\mathrm{IgG})$ antibodies coupled to horseradish peroxidase (HRP) (Bio-Rad). Alternatively (in Fig. 1B), TORC1 activity was estimated as the ratio between the phosphorylation on $\mathrm{Thr}^{737}$ of fulllength Sch9-HA 5 compared to the total abundance of Sch9-HA phosphospecific anti-pThr ${ }^{737}$-Sch9 and 12CA5 antibodies, respectively) as previously described (26).

\section{Coimmunoprecipitation}

Yeast cells expressing the indicated fusion proteins were harvested by filtration. Filters were immediately snap-frozen in liquid nitrogen and

\section{Protein purification}

stored at $-80^{\circ} \mathrm{C}$. Cells were resupended in lysis buffer [50 mM Hepes/KOH ( $\mathrm{pH} 7.4$ ), $150 \mathrm{mM} \mathrm{NaCl}, 10 \mathrm{mM} \mathrm{MgCl}, 0.2 \% \mathrm{NP}-40$ (for myc-Iml1) or $0.5 \% \mathrm{NP}-40$ (for Kog 1 HA), protease and phosphatase inhibitor cocktails (Roche)] and lysed with glass beads using the Precellys cell disruptor. Lysates were diluted in the same lysis buffer (for myc-Iml1) or a buffer devoid of NP-40 (for Kog1-HA) and clarified by two successive centrifugations for $10 \mathrm{~min}$ at $13,000 \mathrm{rpm}$. For input samples, aliquots of cleared lysates were concentrated by precipitation with icecold acetone, resuspended in $6 \times$ concentrated loading buffer, and denatured for $10 \mathrm{~min}$ at $95^{\circ} \mathrm{C}$. For coimmunoprecipitations, cleared lysates were incubated for 2 hours at $4^{\circ} \mathrm{C}$ with prewashed $\mathrm{IgG}$ Sepharose beads (GE Healthcare). After three washes with wash buffer (same as lysis buffer for myc-Imll or containing $450 \mathrm{mM}$ $\mathrm{NaCl}$ for $\mathrm{Kog} 1-\mathrm{HA}$ ), beads were resuspended in $6 \times$ concentrated loading buffer and denatured for $10 \mathrm{~min}$ at $95^{\circ} \mathrm{C}$. Inputs $(25 \mu \mathrm{g})$ and pull-down samples $(2000 \mu \mathrm{g}$ for myc-Iml1, $500 \mu \mathrm{g}$ for Kog1-HA, and 125 or $67.5 \mu \mathrm{g}$ for Gtr1-TAP) were analyzed by SDS-PAGE immunoblot with anti-myc (9E10; Santa Cruz Biotechnology), anti-HA (HA.11; Covance), and antiTAP (Open Biosystems) antibodies together with light chain-specific anti-mouse or antirabbit HRP-conjugated antibodies (Jackson ImmunoResearch).

Glutathione $S$-transferase (GST)-Gtr1, GST-Gtr2, GST-Gtr2 (S23L or Q66L), Gtr1-His 6 , GST-Cdc42, and GST-Iml1 ${ }^{\text {GAP }}$ were produced in the Escherichia coli Rosetta strain after induction with $0.5 \mathrm{mM}$ IPTG (isopropyl$\beta$-D-thiogalactopyranoside) for 5 hours at $18^{\circ} \mathrm{C}$ [GST-Gtrl, GST-Gtr2, and GST-Gtr2 (S23L or Q66L) plus Gtr1-His 6 ], $30^{\circ} \mathrm{C}\left(\mathrm{GST}-\mathrm{Iml} 1^{\mathrm{GAP}}\right)$, or $37^{\circ} \mathrm{C}$ (GST-Cdc42). Cells were collected by centrifugation and lysed with a microfluidizer. Protein fusions were purified with glutathione-Sepharose beads (GE Healthcare), which were washed with buffer A [50 mM Tris$\mathrm{HCl}(\mathrm{pH} 7.5), 200 \mathrm{mM} \mathrm{NaCl}, 1.5 \mathrm{mM} \mathrm{MgCl} 2,5 \%$ glycerol, $1 \mathrm{mM}$ dithiothreitol (DTT), 0.1\% NP-40, and $0.1 \mathrm{mM}$ GDP] for GST-Gtr1, GST-Gtr2, GST-Gtr2 (S23L or Q66L) plus Gtr1-His 6 , and GST-Cdc42, or buffer B [50 mM Tris- $\mathrm{HCl}$ (pH 7.5), $500 \mathrm{mM} \mathrm{NaCl}, 1.5 \mathrm{mM} \mathrm{MgCl}, 5 \%$ glycerol, $1 \mathrm{mM}$ DTT, $1 \%$ Triton X-100, and 0.1\% Tween 20] for GST$\mathrm{Iml1}{ }^{\mathrm{GAP}}$. Proteins were eluted with buffer A plus $10 \mathrm{mM}$ reduced glutathione (without GDP in the case of GST-Iml1 ${ }^{\mathrm{GAP}}$ ). Glycerol was added to a final concentration of $20 \%$. Proteins were snap-frozen in liquid nitrogen and stored at $-80^{\circ} \mathrm{C}$. For the purification of full-length Imll and Iml1 ${ }^{\mathrm{R} 943 \mathrm{~A}}$, yeast iml1 $\mathrm{gtr} 1 \Delta$ double-mutant cells expressing Iml1- or $\mathrm{Imll}^{\mathrm{R} 943 \mathrm{~A}}-\mathrm{His}_{6}-\mathrm{TEV}$-(tobacco etch virus)-cleavage-site-ProteinA were grown in synthetic dropout medium overnight, diluted to an $\mathrm{OD}_{600}$ of 0.2 in YPD (yeast extract, peptone, and dextrose), and further grown to an $\mathrm{OD}_{600}$ of 2.0. Cells were then collected by centrifugation and lysed with a planetary micro mill (Pulverisette). Protein fusions were purified with IgG Sepharose beads (GE Healthcare), which were washed with buffer C 
A
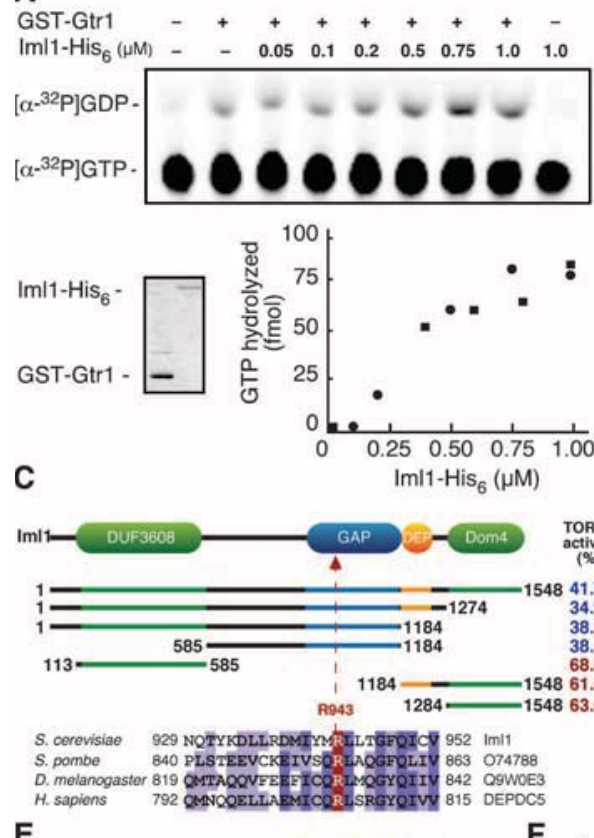

E

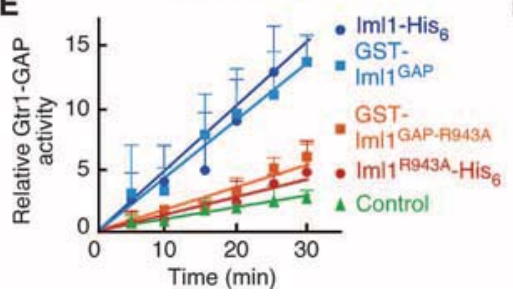

B

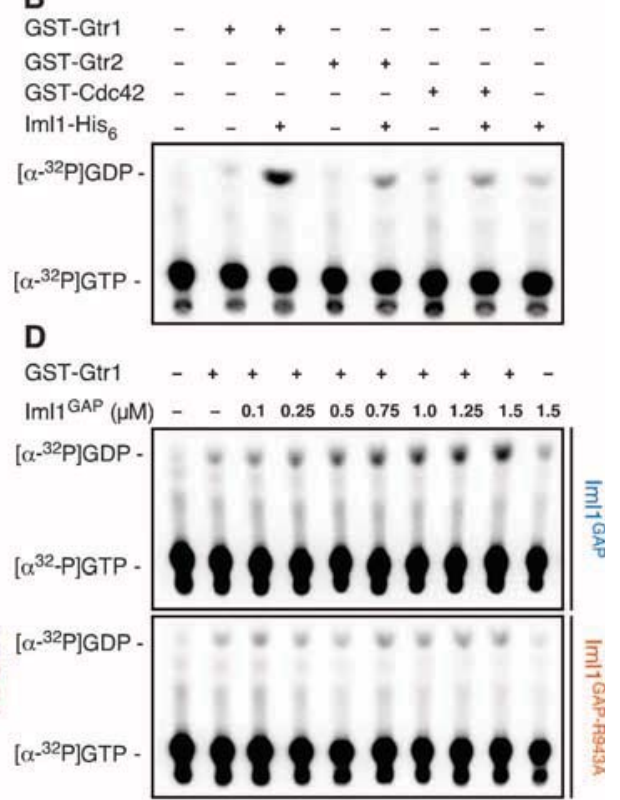

G

\begin{tabular}{|c|c|c|c|c|}
\hline $\begin{array}{c}\text { Fold } \\
\text { activation }\end{array}$ & $\begin{array}{c}\text { Relative GTP } \\
\text { hydrolysis }\end{array}$ & Gtr1 & Gtr2 & $\begin{array}{l}\text { Iml1- } \\
\mathrm{His}_{6}\end{array}$ \\
\hline \multirow{2}{*}{11.3 [ } & $1.0 \pm 0.1$ & + & - & - \\
\hline & $11.3 \pm 1.1$ & + & - & + \\
\hline \multirow{2}{*}{5.3 [ } & $15.9 \pm 0.5$ & + & Q66L & - \\
\hline & $80.6 \pm 7.7$ & + & Q66L & + \\
\hline \multirow{2}{*}{$5.1=[$} & $127.8 \pm 4.6$ & + & S23L & - \\
\hline & $676.9 \pm 13.2$ & + & S23L & + \\
\hline
\end{tabular}

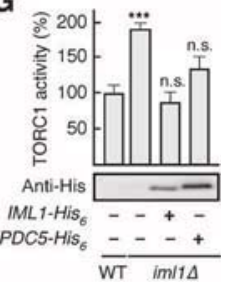

Fig. 4. Iml1 is a GAP for Gtr1. (A) GST-Gtr1 was loaded with [ $\left.\alpha^{-32} P\right]$ GTP, and hydrolysis to [ $\left.\alpha^{-32} P\right]$ GDP was assayed in the absence or presence of increasing concentrations of Iml1-His 6 . Purified proteins were visualized by Coomassie staining (inserted panel), and the results of the GAP assay were quantified and illustrated graphically. One representative thin-layer chromatography (TLC) autoradiograph and the corresponding quantifications from two independent experiments (squares or circles) are shown. (B) Iml1-His 6 activates the intrinsic GTPase activity of Gtr1 to a greater extent than that of Gtr2 or the Rho GTPase Cdc42. One representative TLC autoradiograph from two independent experiments is shown. (C) Schematic representation of the conserved domains within Saccharomyces cerevisiae Iml1 and functional analysis of the TORC1 inhibitory activity of the indicated truncated Iml1 variants. The corresponding constructs were overexpressed in WT cells, and TORC1 activities were normalized to the samples containing the empty vector. Red arrow indicates the position of a conserved arginine within the Iml1 GAP domain that was aligned, together with its flanking residues, with the corresponding amino acid sequences of Iml1 orthologs in higher eukaryotes. Data are means \pm SD of three independent

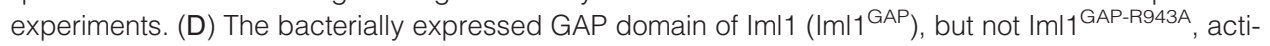
vates the GTPase activity of Gtr1. One representative TLC autoradiograph from three independent experiments is shown. (E) Single-turnover GAP assays on Gtr1 with or without (control) the indicated fulllength Iml1 or Iml1 ${ }^{\text {GAP }}$ variants. Data are means \pm SD from three independent experiments. (F) Relative GTP hydrolysis ( \pm SD; $n=3$ independent experiments) by the indicated combinations of Gtr GTPases (with or without Iml1-His 6 ; normalized to the one of Gtr1 without Iml1-His 6 ). (G) Human DEPDC5 partially complements the TORC1 inhibition defect in im/1 $1 \Delta$ cells. TORC1 activities were assayed as in Fig. 1A. Fusion protein expression was confirmed by immunoblot analysis. ${ }^{\star \star \star} P<0.001$; n.s., not significant, compared to the respective WT control using Student's $t$ test ( $P$ values are Holm-Bonferroni-adjusted). Data are means \pm SD from three independent experiments.

[20 mM Hepes (pH 7.4), $300 \mathrm{mM} \mathrm{NaCl}, 110 \mathrm{mM}$ potassium acetate (KOAc), $2 \mathrm{mM} \mathrm{MgCl}_{2}, 0.1 \%$ Tween 20, $1 \%$ Triton X-100, $1 \mathrm{mM}$ DTT] (15). After overnight TEV cleavage, a second purification step was performed with

Ni-NTA agarose beads (Qiagen), which were washed with buffer $\mathrm{C}$ plus $10 \mathrm{mM}$ imidazole. Proteins were eluted with buffer D [20 mM Hepes (pH 7.4), $75 \mathrm{mM}$ $\mathrm{NaCl}, 110 \mathrm{mM}$ KOAc, $2 \mathrm{mM} \mathrm{MgCl}$, and $300 \mathrm{mM}$ imidazole]. Glycerol was added to a final concentration of $20 \%$. Proteins were snap-frozen in liquid nitrogen and stored at $-80^{\circ} \mathrm{C}$

\section{In vitro Gtr1-Iml1 binding assays}

Purified GST-Gtrl or GST alone (100 nM final) was incubated for $30 \mathrm{~min}$ at room temperature in loading buffer $[20 \mathrm{mM}$ Tris-HCl (pH 8.0), 2 mM EDTA, and $1 \mathrm{mM}$ DTT] in the presence of either GTP $\gamma \mathrm{S}$ (100 $\mu \mathrm{M}$ final), GTP (1 mM final), GDP (1 $\mathrm{mM}$ final), or GDP $+\mathrm{AlF}_{\mathrm{x}}$ (1 $\mathrm{mM}$ GDP, $2 \mathrm{mM} \mathrm{AlCl}_{3}$, and $20 \mathrm{mM} \mathrm{NaF}$ final concentration). Subsequently, purified Iml1$\mathrm{His}_{6}(100 \mathrm{nM})$ and $\mathrm{MgCl}_{2}(10 \mathrm{mM})$ were added, and the mix was incubated for 1 hour at $4^{\circ} \mathrm{C}$. Pull-down experiments were performed with glutathione-Sepharose beads (Qiagen), which were washed with loading buffer containing $10 \mathrm{mM} \mathrm{MgCl}_{2}$ (or $10 \mathrm{mM} \mathrm{MgCl}_{2}, 2 \mathrm{mM} \mathrm{AlCl}$, and 20 $\mathrm{mM} \mathrm{NaF}$; GDP plus $\mathrm{AlF}_{\mathrm{x}}$ ). Finally, beads were resuspended in $2 \times$ Laemmli buffer and boiled, and the supernatants were used for SDS-PAGE immunoblot analyses.

\section{GTP hydrolysis assays}

GAP assays were performed essentially as previously described $(27,28)$. Briefly, GTPases (100 nM) were incubated for $30 \mathrm{~min}$ at room temperature in loading buffer [20 mM Tris- $\mathrm{HCl}$ (pH 8.0), $2 \mathrm{mM}$ EDTA, and $1 \mathrm{mM} \mathrm{DTT}]$ in the presence of $40 \mathrm{nM}\left[\alpha^{-32} \mathrm{P}\right] \mathrm{GTP}$ (Hartman Analytic, $3000 \mathrm{Ci} / \mathrm{mmol}$ ). Full-length Imll$\mathrm{His}_{6} / \mathrm{Iml1}^{\mathrm{R} 943 \mathrm{~A}}$-His6 or GST-Iml1 GAP/ GST-Iml1 ${ }^{\text {GAP-R943A }}$ was then added to the mixtures (at the indicated concentrations), together with $10 \mathrm{mM} \mathrm{MgCl} 2$, to initialize the reactions. After $20 \mathrm{~min}$ of incubation at room temperature, reactions were stopped by addition of elution buffer (1\% SDS, $25 \mathrm{mM}$ EDTA, $5 \mathrm{mM}$ GDP, and $5 \mathrm{mM} \mathrm{GTP}$ ) and heating for $2 \mathrm{~min}$ at $65^{\circ} \mathrm{C}$. $\left[\alpha-{ }^{32} \mathrm{P}\right]$ GTP and $\left[\alpha-{ }^{32} \mathrm{P}\right]$ GDP were separated by TLC on PEI Cellulose F plates (Merck) with buffer containing $1 \mathrm{M}$ acetic acid and $0.8 \mathrm{M} \mathrm{LiCl}$. Results were visualized with a phosphorimager and quantified with ImageQuant. Single-turnover GAP assays were performed with $\mathrm{Imll}_{-\mathrm{His}}(200 \mathrm{nM})$, $\operatorname{Iml} 1^{\mathrm{R}^{2} 43 \mathrm{~A}}-\mathrm{His}_{6}(200 \mathrm{nM})$, GST-Iml1 ${ }^{\mathrm{GAP}}(1 \mu \mathrm{M})$, or GST-Iml1 ${ }^{\mathrm{GAP}-\mathrm{R} 943 \mathrm{~A}}$ $(1 \mu \mathrm{M})$ as described above, except that $1.7 \mathrm{mM}$ unlabeled GTP was added simultaneously with $\mathrm{MgCl}_{2}$. 


\section{SUPPLEMENTARY MATERIALS}

Fig. S1. Loss of individual SEAC subunits does not substantially affect the growth of cells at $30^{\circ} \mathrm{C}$ or elF2 $\alpha$ phosphorylation after leucine starvation.

Fig. S2. Effects on TORC1 activity of various combinations of individual deletions of SEAC subunit-encoding genes.

Fig. S3. Loss of Iml1-Npr2-Npr3 complex subunits, individually or in combination, or expression of Gtr $1^{\mathrm{Q} 65 \mathrm{~L}}$ or of Iml1 $1^{\mathrm{R943A}}$ renders TORC1 activity partially insensitive to leucine starvation.

Fig. S4. Iml1 physically interacts with Gtr1-TAP in the presence, but substantially less in the absence, of Npr2 and Npr3.

Fig. S5. The conserved residue $\mathrm{Arg}^{943}$ in Iml1 is functionally important.

Fig. S6. Purified Iml1-His ${ }_{6}$ and $I \mathrm{III}^{\mathrm{R} 943 \mathrm{~A}}-\mathrm{His}_{6}$ preferentially bind bacterially expressed GST-Gtr1 preloaded with GDP plus AlF $_{\mathrm{x}}$.

Table S1. Strains used in this study.

Table S2. Plasmids used in this study.

References (30) to (34)

\section{REFERENCES AND NOTES}

1. A. Soulard, A. Cohen, M. N. Hall, TOR signaling in invertebrates. Curr. Opin. Cell Biol. 21, 825-836 (2009).

2. R. Zoncu, A. Efeyan, D. M. Sabatini, mTOR: From growth signal integration to cancer, diabetes and ageing. Nat. Rev. Mol. Cell Biol. 12, 21-35 (2011).

3. C. De Virgilio, The essence of yeast quiescence. FEMS Microbiol. Rev. 36, 306-339 (2012).

4. R. Loewith, M. N. Hall, Target of rapamycin (TOR) in nutrient signaling and growth control. Genetics 189, 1177-1201 (2011).

5. E. Kim, P. Goraksha-Hicks, L. Li, T. P. Neufeld, K. L. Guan, Regulation of TORC1 by Rag GTPases in nutrient response. Nat. Cell Biol. 10, 935-945 (2008).

6. M. Binda, M. P. Péli-Gulli, G. Bonfils, N. Panchaud, J. Urban, T. W. Sturgill, R. Loewith, C. De Virgilio, The Vam6 GEF controls TORC1 by activating the EGO complex. Mol. Cell 35, 563-573 (2009).

7. Y. Sancak, T. R. Peterson, Y. D. Shaul, R. A. Lindquist, C. C. Thoreen, L. Bar-Peled, D. M. Sabatini, The Rag GTPases bind raptor and mediate amino acid signaling to mTORC1. Science 320, 1496-1501 (2008).

8. R. Zoncu, L. Bar-Peled, A. Efeyan, S. Wang, Y. Sancak, D. M. Sabatini, mTORC1 senses lysosomal amino acids through an inside-out mechanism that requires the vacuolar $\mathrm{H}^{+}$-ATPase. Science 334, 678-683 (2011).

9. L. Bar-Peled, L. D. Schweitzer, R. Zoncu, D. M. Sabatini, Ragulator is a GEF for the rag GTPases that signal amino acid levels to mTORC1. Cell 150, 1196-1208 (2012).

10. Y. Sancak, L. Bar-Peled, R. Zoncu, A. L. Markhard, S. Nada, D. M. Sabatini, RagulatorRag complex targets mTORC1 to the lysosomal surface and is necessary for its activation by amino acids. Cell 141, 290-303 (2010).

11. N. Valbuena, K. L. Guan, S. Moreno, The Vam6 and Gtr1-Gtr2 pathway activates TORC1 in response to amino acids in fission yeast. J. Cell Sci. 125, 1920-1928 (2012).

12. G. Bonfils, M. Jaquenoud, S. Bontron, C. Ostrowicz, C. Ungermann, C. De Virgilio, Leucyl-tRNA synthetase controls TORC1 via the EGO complex. Mol. Cell 46, 105-110 (2012).

13. J. M. Han, S. J. Jeong, M. C. Park, G. Kim, N. H. Kwon, H. K. Kim, S. H. Ha, S. H. Ryu, S. Kim, Leucyl-tRNA synthetase is an intracellular leucine sensor for the mTORC1signaling pathway. Cell 149, 410-424 (2012).

14. T. K. Neklesa, R. W. Davis, A genome-wide screen for regulators of TORC1 in response to amino acid starvation reveals a conserved Npr2/3 complex. PLoS Genet. 5 , e1000515 (2009).

15. S. Dokudovskaya, F. Waharte, A. Schlessinger, U. Pieper, D. P. Devos, I. M. Cristea, R. Williams, J. Salamero, B. T. Chait, A. Sali, M. C. Field, M. P. Rout, C. Dargemont, A conserved coatomer-related complex containing Sec13 and Seh1 dynamically associates with the vacuole in Saccharomyces cerevisiae. Mol. Cell. Proteomics 10, M110.006478 (2011)

16. X. Wu, B. P. Tu, Selective regulation of autophagy by the Iml1-Npr2-Npr3 complex in the absence of nitrogen starvation. Mol. Biol.Cell 22, 4124-4133 (2011).

17. A. G. Hinnebusch, Translational regulation of GCN4 and the general amino acid control of yeast. Annu. Rev. Microbiol. 59, 407-450 (2005).

18. F. Dubouloz, O. Deloche, V. Wanke, E. Cameroni, C. De Virgilio, The TOR and EGO protein complexes orchestrate microautophagy in yeast. Mol. Cell 19, 15-26 (2005).

19. A. Wittinghofer, I. R. Vetter, Structure-function relationships of the G domain, a canonical switch motif. Annu. Rev. Biochem. 80, 943-971 (2011).
20. K. Scheffzek, M. R. Ahmadian, A. Wittinghofer, GTPase-activating proteins: Helping hands to complement an active site. Trends Biochem. Sci. 23, 257-262 (1998).

21. J. Li, F. Wang, K. Haraldson, A. Protopopov, F. M. Duh, L. Geil, I. Kuzmin, J. D. Minna, E. Stanbridge, E. Braga, V. I. Kashuba, G. Klein, M. I. Lerman, E. R. Zabarovsky, Functional characterization of the candidate tumor suppressor gene NPRL2/G21 located in 3p21.3C. Cancer Res. 64, 6438-6443 (2004).

22. L Ji, M. Nishizaki, B. Gao, D. Burbee, M. Kondo, C. Kamibayashi, K Xu, N. Yen, E. N. Atkinson, B. Fang, M. I. Lerman, J. A. Roth, J. D. Minna, Expression of severa genes in the human chromosome 3p21.3 homozygous deletion region by an adenovirus vector results in tumor suppressor activities in vitro and in vivo. Cancer Res. 62 , 2715-2720 (2002).

23. M. I. Lerman, J. D. Minna, The $630-\mathrm{kb}$ lung cancer homozygous deletion region on human chromosome 3p21.3: Identification and evaluation of the resident candidate tumor suppressor genes. The International Lung Cancer Chromosome 3p21.3 Tumor Suppressor Gene Consortium. Cancer Res. 60, 6116-6133 (2000).

24. T. J. Seng, K. Ichimura, L. Liu, O. Tingby, D. M. Pearson, V. P. Collins, Complex chromosome 22 rearrangements in astrocytic tumors identified using microsatellite and chromosome 22 tile path array analysis. Genes Chromosomes Cancer 43, 181-193 (2005).

25. J. Urban, A. Soulard, A. Huber, S. Lippman, D. Mukhopadhyay, O. Deloche, V. Wanke, D. Anrather, G. Ammerer, H. Riezman, J. R. Broach, C. De Virgilio, M. N. Hall, R. Loewith, Sch9 is a major target of TORC1 in Saccharomyces cerevisiae. Mol. Cell 26, 663-674 (2007).

26. T. Takahara, T. Maeda, Transient sequestration of TORC1 into stress granules during heat stress. Mol. Cell 47, 242-252 (2012).

27. D. H. Anderson, M. D. Chamberlain, Assay and stimulation of the Rab5 GTPase by the p85 $\alpha$ subunit of phosphatidylinositol 3-kinase. Methods Enzymol. 403, 552-561 (2005).

28. M. D. Chamberlain, T. R. Berry, M. C. Pastor, D. H. Anderson, The p85 $\alpha$ subunit of phosphatidylinositol 3'-kinase binds to and stimulates the GTPase activity of Rab proteins. J. Biol. Chem. 279, 48607-48614 (2004).

29. M. K. Sung, W. K. Huh, Bimolecular fluorescence complementation analysis system for in vivo detection of protein-protein interaction in Saccharomyces cerevisiae. Yeast 24, 767-775 (2007).

30. I. Pedruzzi, F. Dubouloz E Cameroni, V. Wanke, J. Roosen, J. Winderickx C. De Virgilio, TOR and PKA signaling pathways converge on the protein kinase Rim15 to control entry into $G_{0}$. Mol. Cell 12, 1607-1613 (2003).

31. C. B. Brachmann, A. Davies, G. J. Cost, E. Caputo, J. Li, P. Hieter, J. D. Boeke, Designer deletion strains derived from Saccharomyces cerevisiae S288C: A useful set of strains and plasmids for PCR-mediated gene disruption and other applications. Yeast 14, 115-132 (1998).

32. R. D. Gietz, A. Sugino, New yeast-Escherichia coli shuttle vectors constructed with in vitro mutagenized yeast genes lacking six-base pair restriction sites. Gene 74, 527-534 (1988).

33. D. Kressler, G. Bange, Y. Ogawa, G. Stjepanovic, B. Bradatsch, D. Pratte, S. Amlacher, D. Strauß, Y. Yoneda, J. Katahira, I. Sinning, E. Hurt, Synchronizing nuclear import of ribosomal proteins with ribosome assembly. Science 338, 666-671 (2012).

34. T. W. Christianson, R. S. Sikorski, M. Dante, J. H. Shero, P. Hieter, Multifunctional yeast high-copy-number shuttle vectors. Gene 110, 119-122 (1992).

Acknowledgments: We thank F. Jaquier and M. Jaquenoud for technical assistance, T. Maeda for anti-pThr ${ }^{73}$-Sch9 antibodies, D. Kressler and R. Loewith for plasmids, and L.-F. Bersier and S. Bacher for advice regarding statistical analyses. Funding: This research was supported by the Canton of Fribourg and the Swiss National Science Foundation (C.D.V.). Author contributions: N.P. and M.-P.P.-G. performed the experiments. All authors contributed to the experimental design and data analysis and commented on the manuscript, which was written by C.D.V. Competing interests: The authors declare that they have no competing interests. 